\title{
Remarks on measurable Boolean algebras and sequential cardinals
}

\author{
by
}

Grzegorz P lebanek (Wrocław)

\begin{abstract}
The paper offers a generalization of Kalton-Roberts' theorem on uniformly exhaustive Maharam's submeasures to the case of arbitrary sequentially continuous functionals. Applying the result one can reduce the problem of measurability of sequential cardinals to the question whether sequentially continuous functionals are uniformly exhaustive.
\end{abstract}

1. Introduction. The celebrated control measure problem posed by D. Maharam amounts to asking whether a $\sigma$-complete Boolean algebra $\mathbf{A}$ admits a measure provided it carries a strictly positive continuous submeasure. A result due to Kalton and Roberts [6] reduced the problem to the question whether every continuous submeasure is uniformly exhaustive (see also Talagrand [13] and Louveau [9]). Fremlin's article on measurable algebras [5] contains a survey on the topic and further references.

We treat here a larger class of functionals in the context of characterizing measurable algebras. The approach presented below is a result of an attempt at solving the following problem stated by Keisler and Tarski [7].

Given $\kappa$ less than the first real-valued measurable cardinal, is it true that every real-valued sequentially continuous function on the Cantor cube $2^{\kappa}$ is actually continuous?

The basic idea is to consider sequentially continuous and strictly positive functionals defined on $\sigma$-complete Boolean algebras, in the sequel called Mazur functionals. Extending Kalton and Robert's result, we show in Section 4 that the existence of a Mazur functional on a $\sigma$-complete Boolean algebra yields its measurability provided a certain exhaustivity-type condition is satisfied. Next, developing an idea due to Antonovskiu and Chudnovsky [1], we prove that if there is a sequentially continuous and discontinuous

Research partially supported by the KBN grant 2/1054/91/01. 
function on $2^{\kappa}$ then there exists a $\sigma$-complete proper ideal $\mathcal{H}$ on $\kappa$ such that the algebra $\mathbf{P}(\kappa) / \mathcal{H}$ carries a Mazur functional (Section 6). This result is a consequence of some technical lemmas of Section 5, showing how to construct "better" functionals from arbitrary ones.

If it happened to be true that every sequentially continuous functional on a $\sigma$-complete Boolean algebra is uniformly exhaustive then both the problems mentioned above would have a positive solution. However, it is rather believed that the control measure problem has a negative answer (cf. Fremlin [5]). Professor Fremlin also remarked (in a private letter) that the two problems may differ dramatically: the former is known to have an absolute answer while the latter is likely to be independent of the usual axioms of set theory.

2. Preliminaries. As regards terminology concerning Boolean algebras and functionals defined on them we essentially follow Fremlin [5]. By a functional on an algebra $\mathbf{A}$ we mean any function $\varphi: \mathbf{A} \rightarrow \mathbb{R}$ with $\varphi(\mathbf{0})=0$. In fact all the functionals considered are assumed to be non-negative. In the context of Boolean algebras, the term measure is reserved for a countably additive functional $\mu$ which is strictly positive, that is, $\mu(a)=0$ implies $a=\mathbf{0}$. A $\sigma$-complete algebra $\mathbf{A}$ is said to be measurable if it carries a probability measure (to avoid trivialities we assume that all Boolean algebras that appear in the sequel are non-zero).

For a sequence $\left(a_{n}\right)$ in a $\sigma$-complete algebra $\mathbf{A}$, we write $a_{n} \rightarrow a$ if

$$
a=\sum_{n \geq 1} \prod_{k \geq n} a_{k}=\prod_{n \geq 1} \sum_{k \geq n} a_{k} .
$$

If moreover the sequence is increasing (decreasing) we write $a_{n} \uparrow a\left(a_{n} \downarrow a\right.$, respectively).

Definition 2.1. A functional $\varphi$ on $\mathbf{A}$ is called sequentially continuous if $\lim _{n} \varphi\left(a_{n}\right)=\varphi(a)$ whenever $a_{n} \rightarrow a$. A sequentially continuous functional $\varphi$ is a Mazur functional if it is strictly positive, i.e. $\varphi(a)=0$ is equivalent to $a=\mathbf{0}$.

The term "Mazur functional" was used in [1] in a slightly different meaning (see the first part of the proof of Theorem 6.1).

Recall that a Maharam submeasure on an algebra $\mathbf{A}$ is a functional $\varphi$ on A which is

- increasing, i.e. $a \leq b$ implies $\varphi(a) \leq \varphi(b)$;

- subadditive, i.e. $\varphi(a+b) \leq \varphi(a)+\varphi(b)$; and

- continuous at $\mathbf{0}$, i.e. $a_{n} \downarrow \mathbf{0}$ implies $\lim _{n} \varphi\left(a_{n}\right)=0$.

Note that if $\varphi$ is a Maharam submeasure then $\varphi$ is sequentially continuous. Indeed, if $a_{n} \downarrow a$ then $\varphi(a) \leq \varphi\left(a_{n}\right) \leq \varphi(a)+\varphi\left(a_{n}-a\right)$, so 
$\lim _{n} \varphi\left(a_{n}\right)=\varphi(a)$, and similarly for $a_{n} \uparrow a$. Thus, if $a_{n} \rightarrow a$ then

$$
\limsup _{k} \varphi\left(a_{k}\right) \leq \lim _{n} \varphi\left(\sum_{k \geq n} a_{k}\right)=\varphi(a)=\lim _{n} \varphi\left(\prod_{k \geq n} a_{k}\right) \leq \liminf _{k} \varphi\left(a_{k}\right),
$$

so $\lim _{n} \varphi\left(a_{n}\right)=\varphi(a)$.

If a $\sigma$-complete algebra $\mathbf{A}$ carries a strictly positive Maharam submeasure then A satisfies the countable chain condition, is complete and weakly $\omega$ distributive (Fremlin [5], Proposition 5.6). The same holds if we assume that $\mathbf{A}$ carries a Mazur functional (the proof is similar).

Let $\varphi$ be a functional on $\mathbf{A}$. We shall often consider functionals $\varphi^{a}$ and $\varphi_{a}$ on $\mathbf{A}$ (for fixed $a \in \mathbf{A}$ ) defined as

$$
\varphi^{a}(c)=\max (\varphi(c+a)-\varphi(a), 0) \quad \text { and } \quad \varphi_{a}(c)=\varphi(c \cdot a) .
$$

Clearly if $\varphi$ is sequentially continuous then so are $\varphi^{a}$ and $\varphi_{a}$.

Recall that a functional $\varphi$ on $\mathbf{A}$ is uniformly exhaustive if for every $\varepsilon>0$ there is an $N$ such that for every family $\mathcal{A}$ of pairwise disjoint elements of A, if $\mathcal{A} \subseteq\{\varphi \geq \varepsilon\}$ then $|\mathcal{A}| \leq N$. We shall consider the following stronger property.

Definition 2.2. Say that a functional $\varphi$ on $\mathbf{A}$ is uniform if for every $a \in \mathbf{A}$ and $\varepsilon>0$ there is an $N$ such that every family of pairwise disjoint elements contained in $\{c: \varphi(c+a) \geq \varphi(a)+\varepsilon\}$ has at most $N$ elements.

In other words, $\varphi$ is uniform if and only if $\varphi^{a}$ is uniformly exhaustive for every $a \in \mathbf{A}$. Every strictly positive uniformly exhaustive Maharam submeasure is thus a uniform Mazur functional.

Given a family $\mathcal{P}$ in an algebra $\mathbf{A}$, the intersection number of $\mathcal{P}$, denoted here by $\operatorname{cal}(\mathcal{P})$, is defined as

$$
\operatorname{cal}(\mathcal{P})=\inf \left\{\#\left(a_{1}, \ldots, a_{n}\right): n \geq 1, a_{i} \in \mathcal{P}\right\},
$$

where

$$
\#\left(a_{1}, \ldots, a_{n}\right)=\frac{1}{n} \max \left\{|I|: I \subseteq\{1, \ldots, n\}, \prod_{i \in I} a_{i} \neq \mathbf{0}\right\} .
$$

The following classical result is due to Kelley [8].

Theorem 2.3. For a family $\mathcal{P} \subseteq \mathbf{A}$ the condition $\mathbf{c a l}(\mathcal{P}) \geq r$ is necessary and sufficient for the existence of an additive functional $\xi$ on $\mathbf{A}$ with $\xi(\mathbf{1})$ $=1$ and $\xi_{\mid \mathcal{P}} \geq r$, i.e. $\xi(a) \geq r$ for all $a \in \mathcal{P}$.

We end this section with a well-known lemma (see e.g. [5], Proposition 5.7). 
LEMMA 2.4. If $\xi$ is an additive functional on an algebra $\mathbf{A}$ then there is a countably additive functional $\mu$ on $\mathbf{A}$ such that $\mu \leq \xi$ and

$$
\mu(\mathbf{1})=\inf \left\{\sup _{n \geq 1} \xi\left(a_{n}\right): a_{n} \uparrow \mathbf{1}\right\} .
$$

3. Key lemma. In this section we prove a technical result (Lemma 3.3) which describes how to construct countably additive functionals on Boolean algebras from arbitrary sequentially continuous ones. We base on the same combinatorial background that was used by Kalton and Roberts in [6] (see also Louveau [9]).

Lemma 3.1 provides a combinatorial tool for evaluating intersection numbers. Although it was not stated as a separate item, its proof can be easily extracted from the first part of the proof of Lemma 3.1 of [6] (cf. [5], the proof of Theorem 5.11). We reproduce the argument for the reader's convenience.

LEMMA 3.1. Suppose that $a_{1}, \ldots, a_{m}$ are elements of $\mathbf{A}$ such that $\#\left(a_{1}, \ldots, a_{m}\right)=q / m$. Given $p$ with $p^{2} \geq 18 m q$, there exist $b_{i j} \in \mathbf{A}, i \leq m$, $j \leq p$, such that

(a) for every $j \leq p, b_{1 j}, \ldots, b_{m j}$ are pairwise disjoint;

(b) for every $i \leq m$ the sequence $b_{i 1}, \ldots, b_{i p}$ contains at most three nonzero elements and $b_{i 1}+\ldots+b_{i p}=a_{i}$.

Proof. We start by recalling two auxiliary facts. Part (1), a lemma on the existence of the so-called concentrator, is taken from [5], Lemma 5.8 (cf. [6]). Part (2) is a consequence of (1) obtained by use of Hall's Marriage Theorem (see e.g. [2], Theorem 3.3).

(1) Suppose that $q, p, m$ are natural numbers such that $1 \leq q \leq m$ and $18 m q \leq p^{2}$. Then there is a set $R \subseteq\{1, \ldots, m\} \times\{1, \ldots, p\}$ such that every vertical section of $R$ has just three members, and $|R[E]| \geq|E|$ for every subset $E$ of $\{1, \ldots, m\}$ having at most $q$ elements. Here $R[E]$ denotes the image of $E$ under $R$, i.e. $R[E]=\{j: j \leq p$, and $(i, j) \in R$ for some $i \in E\}$. (Although such a lemma is proved in [5] under the additional assumption $q \geq 3$, the case $q \leq 3$ is trivial.)

(2) By Hall's Marriage Theorem, for each set $E \subseteq\{1, \ldots, m\}$ with $|E| \leq$ $q$ there is an injective function $f_{E}: E \rightarrow\{1, \ldots, p\}$ such that $(i, f(i)) \in R$ for every $i \in E$.

(3) Now the proof of the assertion of Lemma 3.1 follows. For every $E \subseteq\{1, \ldots, m\}$ we put $b_{E}=\prod_{i \in E} a_{i} \cdot \prod_{i \notin E}\left(-a_{i}\right)$. These are the atoms of the finite algebra generated by $a_{i}$ 's. Note that $b_{E}=\mathbf{0}$ whenever $|E|>q$ (since $\left.\#\left(a_{1}, \ldots, a_{m}\right)=q / m\right)$. For every $i \leq m$ and $j \leq p$ we put

$$
b_{i j}=\sum\left\{b_{E}: i \in E, E \subseteq\{1, \ldots, m\},|E| \leq q, f_{E}(i)=j\right\} .
$$

We shall check that $b_{i j}$ 's are as required. 
The remark above explains that $b_{i 1}+\ldots+b_{i p}=a_{i}$ for every $i \leq m$. Moreover, $b_{i j}=\mathbf{0}$ whenever $(i, j) \notin R$, so there are at most three non-zero elements among $b_{i 1}, \ldots, b_{i p}$.

If $b_{i_{1} j} \cdot b_{i_{2} j} \neq \mathbf{0}$ then there is an $E \subseteq\{1, \ldots, m\}$ with $|E| \leq q$ such that $i_{1}, i_{2} \in E$ and $f_{E}\left(i_{1}\right)=f_{E}\left(i_{2}\right)$. This means $i_{1}=i_{2}$ since $f_{E}$ is injective, so (a) holds.

Lemma 3.2. Let $\varphi$ be a uniformly exhaustive functional on A. Suppose that

$$
\varepsilon=\inf \left\{\max _{i \leq 4} \varphi\left(c_{i}\right): c_{i} \in \mathbf{A}, c_{1}+\ldots+c_{4}=\mathbf{1}\right\}>0,
$$

and put $\mathcal{P}=\{a \in \mathbf{A}: \varphi(-a)<\varepsilon\}$. Then the family $\mathcal{P}$ has a positive intersection number.

Proof. Suppose otherwise; then for a given $\delta>0$ there are a natural number $m$ and $a_{1}, \ldots, a_{m}$ in $\mathcal{P}$ with $\#\left(a_{1}, \ldots, a_{m}\right)=q / m \leq \delta$. Let $p$ be a natural number with $p^{2} \geq 18 m q \geq(p-1)^{2}$, and let $b_{i j}$ be as in the previous lemma. It follows that for every $i \leq m$ there is a $g(i) \leq p$ with $\varphi\left(b_{i g(i)}\right) \geq \varepsilon$. Take $j^{*}$ such that the set $E=\left\{i \leq m: g(i)=j^{*}\right\}$ has at least $m / p$ elements. Now $\left\{b_{i j^{*}}: i \in E\right\}$ is a sequence of pairwise disjoint elements on which $\varphi$ is not less than $\varepsilon$. Since

$$
|E| \geq m / p \geq m /(\sqrt{18 m q}+1) \geq m /(10 \sqrt{m q}) \geq 1 /(10 \sqrt{\delta}),
$$

and $\delta$ can be taken arbitrarily small, it follows that $\varphi$ is not uniformly exhaustive, a contradiction.

LEMMA 3.3. Let $\varphi$ be a non-trivial sequentially continuous functional on a $\sigma$-complete algebra $\mathbf{A}$ with the following properties:

(a) $\varphi$ is uniformly exhaustive;

(b) $\varepsilon=\inf \left\{\max _{i \leq 4} \varphi\left(c_{i}\right): c_{i} \in \mathbf{A}, c_{1}+\ldots+c_{4}=\mathbf{1}\right\}>0$.

Then there exists a non-trivial countably additive functional $\mu$ on $\mathbf{A}$.

Proof. Put $\mathcal{P}=\{a \in \mathbf{A}: \varphi(-a)<\varepsilon\}$. Applying Lemma 3.2 to $\mathcal{P}$ we get $r=\operatorname{cal}(\mathcal{P})>0$. Let $\xi$ be a probability additive functional on $\mathbf{A}$ with $\xi_{\mid \mathcal{P}} \geq r$ (Theorem 2.3). Let $\mu \leq \xi$ be a countably additive functional as in Lemma 2.4. We are to check that $\mu(\mathbf{1})>0$.

Given a sequence $\left(a_{n}\right)$ in $\mathbf{A}$ such that $a_{n} \uparrow \mathbf{1}$, we have $-a_{n} \downarrow \mathbf{0}$ so $\varphi\left(-a_{n_{0}}\right)<\varepsilon$ for some $n_{0}$. Thus $\xi\left(a_{n_{0}}\right) \geq r$; it follows that $\mu(\mathbf{1}) \geq r>0$ and we are done.

4. On uniform Mazur functionals. We show here that if a $\sigma$-complete algebra admits a uniform Mazur functional then it is measurable. This generalizes results on uniformly exhaustive strictly positive Maharam submeasures (see e.g. Theorem 5.12 in Fremlin [5]). (Recall that every strictly posi- 
tive Maharam submeasure is a Mazur functional, and every such submeasure which is uniformly exhaustive is a uniform Mazur functional.)

It follows that every $\sigma$-complete Boolean algebra carrying a Mazur functional is measurable if and only if every Mazur functional is uniform.

LEMMA 4.1. (a) If $\varphi$ is a uniform functional on $\mathbf{A}$ then $\varphi^{a}$ is uniform for every $a \in \mathbf{A}$.

(b) If $\varphi_{1}, \ldots, \varphi_{k}$ are uniform functionals on $\mathbf{A}$ then so is $\varphi=\max \left(\varphi_{1}, \ldots\right.$ $\left.\ldots, \varphi_{k}\right)$

Proof. Since the inequality $\varphi^{a}(c+b) \geq \varphi^{a}(b)+\varepsilon$ implies that $\varphi(c+$ $b+a) \geq \varphi(b+a)+\varepsilon$, part (a) follows from the definition of uniformity. The proof of (b) is straightforward.

LEMMA 4.2. Let A be a $\sigma$-complete Boolean algebra that carries a uniform Mazur functional $\varphi$. For every natural $k$ there exists a sequentially continuous and uniformly exhaustive functional $\tau$ on $\mathbf{A}$ such that

$$
\varepsilon=\inf \left\{\max _{i \leq k} \tau\left(c_{i}\right): c_{i} \in \mathbf{A}, c_{1}+\ldots+c_{k}=1\right\}>0 .
$$

Proof. This clearly holds for $k=1$. We proceed by induction on $k$. Suppose that the assertion holds for $\tau, \varepsilon$ and $k-1$, but there is no such functional for $k$.

By induction we construct $b_{n i} \in \mathbf{A}, n \geq 1, i \leq k$, with the properties:

- $\sum_{i \leq k} b_{n i}=b_{n-1, k}\left(\right.$ where $\left.b_{0 k}=1\right)$;

- $\tau\left(\sum_{m \leq n} b_{m i}\right) \leq \varepsilon\left(2^{-2}+\ldots+2^{-n-1}\right)$;

- $\varphi\left(b_{n k}\right) \leq \varepsilon 2^{-n-1}$.

Having defined $b_{m i}$ for $m \leq n$ and $i \leq k$, we put $e_{i}=\sum_{m \leq n} b_{m i}$, $i=1, \ldots, k-1$, and consider a new functional $\bar{\tau}$, where

$$
\bar{\tau}(c)=\max \left(\max _{i \leq k-1}\left(\tau\left(e_{i}+c \cdot b_{n k}\right)-\tau\left(e_{i}\right)\right), \varphi\left(b_{n k} \cdot c\right)\right) .
$$

Since $\bar{\tau}$ is uniformly exhaustive by Lemma 4.1 , there are $b_{n+1, i}$ such that $\sum_{i<k} b_{n+1, i}=b_{n k}$ and $\bar{\tau}\left(b_{n+1, i}\right) \leq \varepsilon 2^{-n-2}$, and the induction follows.

Since $\left(b_{n k}\right)_{n \geq 1}$ is a decreasing sequence with $\lim _{n} \varphi\left(b_{n k}\right)=0$ and $\varphi$ is strictly positive, $\prod_{n \geq 1} b_{n k}=\mathbf{0}$. Thus, putting $a_{i}=\sum_{n \geq 1} b_{n i}, i=1, \ldots, k-1$, we have $\sum_{i \leq k-1} a_{i}=\mathbf{1}$. On the other hand, $\tau\left(a_{i}\right) \leq \varepsilon / 2$, a contradiction with our choice of $\tau$.

Theorem 4.3. Let $\mathbf{A}$ be a $\sigma$-complete Boolean algebra. If $\mathbf{A}$ carries a uniform Mazur functional then $\mathbf{A}$ is measurable.

Proof. It follows from Lemmas 4.2 and 3.3 that there exists a non-zero countably additive functional $\mu$ on $\mathbf{A}$. Let $\mathcal{A}$ be a maximal family of pairwise disjoint elements from $\left\{a \in \mathbf{A}^{+}: \mu(a)=0\right\}$. Then $\mathcal{A}$ is countable (as $\mathbf{A}$ is a ccc algebra $)$, so $\mu\left(\sum \mathcal{A}\right)=0$. Putting $a_{0}=-\sum \mathcal{A}$ we infer that $\mu$ is a 
measure on the algebra $\mathbf{A} \mid a_{0}$. It is clear that we can repeat the argument for $\mathbf{A} \mid-a_{0}$. This gives at most countable sequences $\left(a_{n}\right)$ and $\left(\mu_{n}\right)$ such that $\mu_{n}$ is a measure on $\mathbf{A} \mid a_{n}$ and $\sum_{n} a_{n}=1$. Finally, $\mu=\sum_{n} 2^{-n} \mu_{n}$ is a measure on $\mathbf{A}$ and this finishes the proof.

Corollary 4.4. If $\varphi$ is a Mazur functional on a $\sigma$-complete Boolean algebra $\mathbf{A}$ then $\mathbf{A}$ is measurable if and only if $\varphi$ is uniform.

Proof. In view of the theorem above, it remains to check that the condition is necessary. Let $\mu$ be a measure on A. Notice that every sequentially continuous functional $\tau$ on $\mathbf{A}$ is absolutely continuous with respect to $\mu$ (in the usual $\varepsilon-\delta$ sense). Indeed, otherwise there would be $a_{n} \in \mathbf{A}$ such that $\mu\left(a_{n}\right) \leq 2^{-n}$ and $\tau\left(a_{n}\right) \geq \varepsilon$ for some $\varepsilon>0$. Putting $b_{n}=\sum_{k>n} a_{k}$ and $b=\prod_{n \geq 1} b_{n}$ we get $\mu(b)=0$, so $b=\mathbf{0}$. Therefore $a_{n} \rightarrow \mathbf{0}$, a contradiction with sequential continuity of $\tau$. Applying this remark to $\varphi^{a}$ we easily get the uniformity condition for $\varphi$.

5. Sequentially continuous functionals on Boolean algebras. Throughout this section we fix a $\sigma$-complete algebra $\mathbf{A}$ that admits a nonzero sequentially continuous functional. We shall show that on $\mathbf{A}$ there exists a functional $\tau$ such that $\mathcal{H}=\{e: \tau(e)=0\}$ is a $\sigma$-complete proper ideal in $\mathbf{A}$, and the algebra $\mathbf{A} / \mathcal{H}$ carries a Mazur functional. Lemma 5.2 is essentially due to Antonovskiu and Chudnovsky [1]. The form of Lemma 5.3 is motivated by an application in the next section.

LEMMA 5.1. There exists a sequentially continuous functional $\tau$ on $\mathbf{A}$ such that $\tau(\mathbf{1})=1$, with the following property:

$$
\text { if } a \leq z, \tau(a)=0 \text { and } \tau(z)=1 \text { then } \tau(z-a)=1 \text {. }
$$

P r o of. Suppose the contrary, i.e. that for every sequentially continuous functional $\tau$ on $\mathbf{A}$ there are $a \leq z$ with $\tau(a)=0, \tau(z)=1$ and $\tau(z-a)<1$. Let $\varphi$ be a fixed sequentially continuous functional on $\mathbf{A}$ with $\varphi(\mathbf{1})=1$.

We shall construct a pairwise disjoint sequence $\left(a_{\xi}: \xi<\omega_{1}\right) \subseteq \mathbf{A}$ and a decreasing sequence $\left(x_{\xi}: \xi<\omega_{1}\right) \subseteq \mathbf{A}$ such that for every $\xi<\omega_{1}$,

(i) $a_{\xi} \leq x_{\xi}$;

(ii) $\varphi\left(\sum_{\eta<\xi} a_{\eta}\right)=0$;

(iii) $\varphi\left(\sum_{\eta<\xi} a_{\eta}+x_{\xi}\right)=1$;

(iv) $\varphi\left(\sum_{\eta<\xi}^{\eta<\xi} a_{\eta}+\left(x_{\xi}-a_{\xi}\right)\right)<1$.

Having constructed such $a_{\xi}$ and $x_{\xi}$ for $\xi<\alpha$, we put $y=\prod_{\xi<\alpha} x_{\xi}$ and $e=\sum_{\xi<\alpha} a_{\xi}$, and consider a functional $\tau$ on $\mathbf{A}$, where $\tau(c)=\varphi(e+y \cdot c)$.

Let us check that $\tau(\mathbf{1})=1$. Indeed, if $\alpha=\beta+1$ then $y=x_{\beta}$ and

$$
e+x_{\beta}=\sum_{\xi<\beta} a_{\xi}+a_{\beta}+x_{\beta}=\sum_{\xi<\beta} a_{\xi}+x_{\beta},
$$


so $\tau(\mathbf{1})=\varphi\left(e+x_{\beta}\right)=1$ (by (iii)). If $\alpha$ is a limit ordinal then, choosing any cofinal increasing sequence $\alpha_{n}$ in $\alpha$, we have

$$
\tau(\mathbf{1})=\varphi(e+y)=\lim _{n} \varphi\left(\sum_{\xi<\alpha_{n}} a_{\xi}+x_{\alpha_{n}}\right)=1 .
$$

By our assumption $\tau$ does not have property $(*)$ so there are $c, z \in \mathbf{A}$ with $c \leq z, \tau(c)=0, \tau(z)=1$ and $\tau(z-c)<1$. Now it is easy to see that $a_{\alpha}=c \cdot y-e$ and $x_{\alpha}=y \cdot z$ are as required.

Now there is a $\delta>0$ and an increasing sequence $\xi_{n}$ such that

$$
\varphi\left(\sum_{\eta<\xi_{n}} a_{\eta}+\left(x_{\xi_{n}}-a_{\xi_{n}}\right)\right) \leq 1-\delta .
$$

Put $\xi=\sup _{n} \xi_{n}, e=\sum_{\eta<\xi} a_{\eta}$ and $x=\prod_{\eta<\xi} x_{\xi}$. Since

$$
\sum_{\eta<\xi_{n}} a_{\eta}+\left(x_{\xi_{n}}-a_{\xi_{n}}\right) \rightarrow e+x,
$$

we get $\varphi(e+x) \leq 1-\delta$. On the other hand,

$$
\sum_{\eta<\xi_{n}} a_{\eta}+x_{\xi_{n}} \rightarrow e+x
$$

so $\varphi(e+x)=1$, a contradiction.

LEMMA 5.2. There exists a non-zero sequentially continuous functional $\varrho$ on $\mathbf{A}$ such that

(i) $\varrho(b)=0$ whenever $b \leq a$ and $\varrho(a)=0$;

(ii) $\varrho(a+b)=0$ whenever $\varrho(a)=\varrho(b)=0$.

The family $\mathcal{H}=\{e: \varrho(e)=0\}$ is then a $\sigma$-complete proper ideal in $\mathbf{A}$.

Proof. Let $\tau$ be a functional as in Lemma 5.1. Notice first that there is an $x \in \mathbf{A}$ such that $\tau(x)=1$ and

(**) for every $y \leq x$ and $a \leq x-y$, if $\tau(y)=1$ then $\tau(a)=0$.

Indeed, otherwise we can easily define a sequence $\left(a_{\xi}: \xi<\omega_{1}\right) \subseteq \mathbf{A}$ and a decreasing sequence $\left(x_{\xi}: \xi<\omega_{1}\right) \subseteq \mathbf{A}$ with $a_{\xi} \leq x_{\xi}-x_{\xi+1}, \tau\left(x_{\xi}\right)=1$ and $\tau\left(a_{\xi}\right)>0$. There is a $\delta>0$ such that $\tau\left(a_{\xi_{n}}\right) \geq \delta$ for some infinite sequence $\left(\xi_{n}\right)$. Then we have $a_{\xi_{n}} \rightarrow \mathbf{0}$, a contradiction with sequential continuity of $\tau$.

We shall check that $\varrho=\tau_{x}$ satisfies (i) and (ii) (recall that $\tau_{x}$ is given by $\tau_{x}(c)=\tau(c \cdot x)$ ).

To check (i) suppose that $\varrho(a)=0$ and $b \leq a$. Then $\tau(a \cdot x)=0$, so $\tau(x-a)=1$ by $(*)$, and $\varrho(b)=\tau(b \cdot x)=0$ by $(* *)$.

If $a \cdot b=\mathbf{0}$ and $\varrho(a)=\varrho(b)=0$ then $\tau(x-a)=1$ by $(*)$, and $\tau(x-$ $(a+b))=1((*)$ again); consequently, $\varrho(a+b)=\tau(x \cdot(a+b))=0$ by $(* *)$. 
Finally, given any $a, b$ with $\varrho(a)=\varrho(b)=0$, we have $\varrho(a+b)=\varrho((a-$ b) $+b)=0$.

Properties (i) and (ii) mean that $\mathcal{H}$ is an ideal in $\mathbf{A} ; \sigma$-completeness follows immediately from sequential continuity of $\varrho$.

In the sequel, the symbol $\div$ denotes symmetric difference, i.e. $a \div b=$ $a \cdot-b+b \cdot-a$.

Lemma 5.3. Let $\varphi$ be a sequentially continuous functional on $\mathbf{A}$ and let $\mathcal{H}$ be a $\sigma$-complete proper ideal in $\mathbf{A}$ such that $\varphi(e)=0$ and $\varphi(-e)>0$ for all $e \in \mathcal{H}$. There exists a non-zero sequentially continuous functional $\tau$ on A such that $\tau(a)=\tau(b)$ whenever $a \div b \in \mathcal{H}$. In particular, $\tau$ is zero on elements of $\mathcal{H}$. If , moreover, $\mathcal{H}=\{e: \varphi(e)=0\}$, then $\tau$ can be chosen so that $\mathcal{H}=\{e: \tau(e)=0\}$.

Pro of. Notice first that, given $a \in \mathbf{A}$, there is a $b_{a} \leq a$ such that $a-b_{a} \in$ $\mathcal{H}$ and for every $c \leq b_{a}$, if $b_{a}-c \in \mathcal{H}$ then $\varphi\left(b_{a}\right)=\varphi(c)$. Otherwise there exists a decreasing sequence $\left(b_{\xi}: \xi<\omega_{1}\right) \subseteq \mathbf{A}$ satisfying, for every $\xi<\omega_{1}$, $a-b_{\xi} \in \mathcal{H}$ and $\varphi\left(b_{\xi}\right) \neq \varphi\left(b_{\xi+1}\right)$. Choose $\delta>0$ and an increasing sequence of $\xi_{n}$ 's such that $\left|\varphi\left(b_{\xi_{n}}\right)-\varphi\left(b_{\xi_{n+1}}\right)\right| \geq \delta$. Since $\prod_{n} b_{\xi_{n}}=\prod_{n+1} b_{\xi_{n+1}}$, this contradicts the sequential continuity.

We define $\tau(a)=\varphi\left(b_{a}\right)$. If $b_{a}^{\prime}$ has the same property as $b_{a}$ then

$$
\varphi\left(b_{a}\right)=\varphi\left(b_{a} \cdot b_{a}^{\prime}\right)=\varphi\left(b_{a}^{\prime}\right),
$$

so the definition is correct. If $a_{1} \div a_{2} \in \mathcal{H}$ then $\tau\left(a_{1}\right)=\varphi\left(b_{a_{1}} \cdot b_{a_{2}}\right)=\tau\left(a_{2}\right)$. It remains to check that $\tau$ is sequentially continuous.

Let $a_{n} \rightarrow a$ and put $e=\sum_{n}\left(a_{n}-b_{a_{n}}\right)$. Then $b_{a_{n}}-e=a_{n}-e \rightarrow a-e$, so

$$
\tau\left(a_{n}\right)=\varphi\left(b_{a_{n}}\right)=\varphi\left(b_{a_{n}}-e\right) \rightarrow \varphi(a-e) .
$$

Let $b=a-e$. Given $c \leq b$ with $b-c \in \mathcal{H}$, we let $f=e+(b-c)(\in \mathcal{H})$. Now

$$
\varphi(c)=\varphi(a-f)=\lim _{n} \varphi\left(b_{a_{n}}-f\right)=\lim _{n} \varphi\left(b_{a_{n}}\right)=\varphi(a-e)=\varphi(b) .
$$

This proves that $\tau(a)=\varphi(b)$, and the continuity follows.

It is clear that $\tau(a)=0$ whenever $a \in \mathcal{H}$. If $\mathcal{H}=\{a: \varphi(a)=0\}$ then $\tau(a)=0$ means $\varphi\left(b_{a}\right)=0$ and $\varphi\left(a-b_{a}\right)=0$; consequently, $a=b_{a}+\left(a-b_{a}\right) \in$ $\mathcal{H}$ and $\varphi(a)=0$.

Proposition 5.4. If a $\sigma$-complete algebra $\mathbf{A}$ carries a non-zero sequentially continuous functional then it carries such a functional $\tau$ with the additional properties that $\tau\left(a_{1}\right)=\tau\left(a_{2}\right)$ whenever $\tau\left(a_{1} \div a_{2}\right)=0$, and $\tau(b)=0$ whenever $b \leq a$ and $\tau(a)=0$. Consequently, there is a proper $\sigma$-complete ideal $\mathcal{H}$ in $\mathbf{A}$ such that $\mathbf{A} / \mathcal{H}$ carries a Mazur functional.

Proof. The first assertion follows from 5.2 and 5.3. We put

$$
\mathcal{H}=\{a \in \mathbf{A}: \tau(a)=0\} ;
$$


it is easy to check that the formula $\tau^{\prime}(\pi(a))=\tau(a)$, where $\pi: \mathbf{A} \rightarrow \mathbf{A} / \mathcal{H}$ is the canonical epimorphism, defines a Mazur functional on $\mathbf{A} / \mathcal{H}$.

6. On measurability of sequential cardinals. Suppose that $\mu$ is a probability measure defined for all subsets of $\kappa$ and vanishing on countable sets. Identifying the power set $\mathbf{P}(\kappa)$ of $\kappa$ with the Cantor cube, we may regard $\mu$ to be a function $\mu: 2^{\kappa} \rightarrow[0,1]$. It is not difficult to check that $\mu$ is sequentially continuous; as $\mu$ is zero on a dense subset, it is not continuous.

Following [1] we say that $\kappa$ is a sequential cardinal if there exists a realvalued sequentially continuous but not continuous function on $2^{\kappa}$. Note that if $\kappa$ is sequential and $\lambda \geq \kappa$ then so is $\lambda$. Denote by $\kappa_{0}$ the least sequential cardinal in case such cardinals exist. The remark above explains that $\kappa_{0}$ is less than or equal to the first real-valued measurable cardinal. Keisler and Tarski [7] posed the problem, which to my best knowledge is still open, whether $\kappa_{0}$ is actually equal to the latter cardinal. We mention below a partial answer given by Antonovskiı and Chudnovsky.

Such considerations were originated by Mazur [10] in connection with the problem of the continuity of functions defined on products of separable metric spaces (see also Engelking [3], Antonovskiu--Chudnovsky [1] and Fremlin [4]). Sequentiality of $\kappa$ is also related to some properties of the Banach space $C\left(2^{\kappa}\right)$ (see [11] and [12]).

THEOREM 6.1. If $\kappa$ is a sequential cardinal then there exists a $\sigma$-complete proper ideal $\mathcal{H}$ in $\mathbf{P}(\kappa)$ containing all singletons and such that the algebra $\mathbf{B}=\mathbf{P}(\kappa) / \mathcal{H}$ carries a Mazur functional.

Proof. (1) Let $g: 2^{\kappa} \rightarrow \mathbb{R}$ be sequentially continuous but not continuous. Following Mazur [10] we get a sequentially continuous functional $\varphi$ defined on the algebra $\mathbf{P}(\kappa)$ such that $\varphi(\kappa)>0$ and $\varphi(I)=0$ for all countable $I \subseteq \kappa$. Here is the sketch of the proof: the space

$$
\mathbf{Q}=\{I \subseteq \kappa:|I| \leq \omega\} \subseteq 2^{\kappa}
$$

is sequential, that is, closure in $\mathbf{Q}$ is determined by ordinary sequences; moreover, $2^{\kappa}$ is the Cech-Stone compactification of $\mathbf{Q}$. Thus $g_{\mid \mathbf{Q}}$ is continuous; denoting by $f$ the unique extension of $g_{\mid \mathbf{Q}}$ to $2^{\kappa}$ we take $\varphi=|f-g|$.

(2) It follows from Lemma 5.1 (or can be easily checked) that we may additionally require $\varphi$ to satisfy $\varphi(\kappa-I)>0$ for all countable $I$. (Actually, if we want to make use of Lemma 5.1 here, a brief analysis of its proof is necessary to make sure that a new function still vanishes on countable sets.)

(3) By Lemma 5.3, applied to the ideal $\mathcal{I}$ of countable subsets of $\kappa$, we can additionally assume that $|A \div B| \leq \omega$ implies $\varphi(A)=\varphi(B)$. This in fact means that we can consider $\varphi$ to be a non-zero sequentially continuous functional on the $\sigma$-complete algebra $\mathbf{A}=\mathbf{P}(\kappa) / \mathcal{I}$. Now we apply Proposition 5.4 and the proof is complete. 
Corollary 6.2. If it is true that every Mazur functional on a $\sigma$-complete Boolean algebra is uniform (in particular: if every sequentially continuous functional on such an algebra is uniformly exhaustive) then $\kappa_{0}$ is a realvalued measurable cardinal.

Proof. This is a consequence of Theorems 6.1 and 4.3.

Remark. The dichotomy $\kappa_{0} \leq 2^{\omega}$ or $\kappa_{0}$ is a measurable cardinal, discovered by Antonovskiu and Chudnovsky [1], can be easily derived from Theorem 6.1. The same authors showed that under Martin's Axiom $2^{\omega}$ is not sequential. A simpler proof of that result was given by Fremlin [4], Theorem 24.F. There the fact that $\kappa_{0}<2^{\omega}$ contradicts Martin's Axiom is shown by a purely topological argument (under MA the space $2^{\kappa}$ is sequentially separable for every $\left.\kappa<2^{\omega}\right)$. For the fact that $\kappa_{0}>2^{\omega}$ under MA we can argue as follows.

Take a $2^{\omega}$-Luzin set $L \subseteq \mathbb{R}$, that is, $|L|=2^{\omega}$ and $|L \cap D|<2^{\omega}$ for every nowhere dense $D$ (MA implies the existence of such $L$ ). If we suppose that $\kappa_{0}=2^{\omega}$ then we can construct a sequentially continuous functional $\varphi: \mathbf{P}(L) \rightarrow \mathbb{R}$ as in the proof of Theorem 6.1 (with $\varphi(L)=1$ ). Denoting by $\left(q_{n}\right)_{n \geq 1}$ any sequence containing all rational numbers we choose inductively open sets $V_{k}$ such that $q_{k} \in V_{k}$ and

$$
\varphi\left(\left(V_{1} \cup \ldots \cup V_{k}\right) \cap L\right) \leq 2^{-2}+\ldots+2^{-k-1} .
$$

Putting $V=\bigcup_{k \geq 1} V_{k}$ we get $\varphi(V \cap L) \leq 1 / 2$. Now the formula

$$
\tau(C)=\varphi(C \cup(V \cap L))-\varphi(V \cap L)
$$

defines a non-zero sequentially continuous functional on $\mathbf{P}(L-V)$. Since $\tau$ vanishes on countable sets, it is not continuous. As $|L-V|<2^{\omega}$, this is a contradiction.

Acknowledgement. I should like to thank the referee for his several helpful comments that enabled me to correct and improve the former version of this paper.

\section{References}

[1] M. Antonovskiu and D. Chudnovsky, Some questions of general topology and Tikhonov semifields II, Russian Math. Surveys 31 (1976), 69-128.

[2] I. Anderson, A First Course in Combinatorial Mathematics, Clarendon Press, 1974.

[3] R. Engelking, On functions defined on Cartesian products, Fund. Math. 59 (1966), 221-231.

[4] D. H. Fremlin, Consequences of Martin's Axiom, Cambridge Univ. Press, 1984.

[5] —, Measure algebras, in: Handbook of Boolean Algebras, J. D. Monk (ed.), NorthHolland, 1989, Vol. III, Chap. 22. 
[6] N. J. Kalton and J. W. Roberts, Uniformly exhaustive submeasures and nearly additive set functions, Trans. Amer. Math. Soc. 278 (1983), 803-816.

[7] H. J. Keisler and A. Tarski, From accessible to inaccessible cardinals, Fund. Math. 53 (1964), 225-306.

[8] J. K. Kelley, Measures on Boolean algebras, Pacific J. Math. 9 (1959), 1165-1177.

[9] A. Louveau, Progrès récents sur le problème de Maharam d'après N. J. Kalton et J. W. Roberts, Publ. Math. Univ. Pierre Marie Curie 66 (1983/1984).

[10] S. Mazur, On continuous mappings on Cartesian products, Fund. Math. 39 (1952), 229-238.

[11] G. Plebanek, On the space of continuous functions on a dyadic set, Mathematika 38 (1991), 42-49.

[12] -, On the Mazur Property and realcompactness in $C(K)$, in: Topology, Measures and Fractals, Math. Res. 66, Akademie-Verlag, 1992, 27-36.

[13] M. Talagrand, A simple example of pathological submeasure, Math. Ann. 252 (1980), 97-102.

INSTITUTE OF MATHEMATICS

WROCEAW UNIVERSITY

PL. GRUNWALDZKI $2 / 4$

50-384 WROCEAW, POLAND

E-mail: PLEBANEK@PLWRUW11.BITNET

Received 17 March 1992;

in revised form 19 September 1992 and 7 January 1993 